\title{
Application of platelet fibrin as gingival graft: case report
}

\section{Introduction}

Platelet Rich Fibrin (PRF) was developed by Choukroun, and is considered an innovation in oral and maxillofacial surgery, integrates the second generation of platelet concentrates that offers the clot or membrane a large cluster of growth factors. ${ }^{1}$ They are obtained by centrifuging a blood sample of total autogenous origin, without adding external chemicals and / or anticoagulants, which can be used in order to ensure better healing and promote tissue regeneration. ${ }^{2,3}$ Plaquetterich fibrin presents itself as an improved clot, with a conformation that allows the graft to be restored with the patient's own cells, stimulating the natural healing process more quickly. ${ }^{1}$ It is commonly used in patients undergoing extraction, alveolar grafts, maxillary sinus lifting, increased bone tissue for implantology, aesthetic periodontal surgery, among others. ${ }^{4}$ In periodontics the use of PRF has been gaining strength due to the proven periodontal regeneration process, having shown good results in the increase of keratinized gingiva, and for covering gingival recessions. ${ }^{5}$

The aim of the present study is to report a clinical case of using PRF as a substitute for gingival graft.

\section{Methodology}

This work is a case study associated with a literature review, produced through scientific articles indexed in the main databases such as PubMed, Scielo, Lilacs and Bireme and websites.

A 65-year-old individual with Hypertension was selected for the sample. The patient underwent clinical examinations to rule out the possibility of any oral disease present. Root scraping was performed to adapt the medium.

The patient underwent graft surgery, opting for the use of PRF as material.The surgery was performed with an appropriate instrument, previously sterilized according to the recommended standard, the entire surgical team used the individual protection equipment, and the patient signed the free and informed consent form.

\section{Case report}

A 65-year-old female patient, ASA 2 (with arterial hypertension) came to the office complaining of sensitivity and aesthetic discomfort in units 41 and after clinical examination, Miller Class I recession (Figure 1) was observed in the units, and little keratinized tissue in the region. A sub epithelial connective tissue graft procedure was suggested to the patient to cover the region (as it is the standard model), but the patient did not want to undergo two surgical sites. Then the patient was suggested to use L-PRF as a replacement for the subepithelial graft.

To obtain the L-PRF, blood was collected using $9 \mathrm{ml}$ tubes, without adding any additives (VacutubeSeco, Biocon $\AA$, Brazil). The blood used to make the L-PRF was taken from the patient herself, these were placed in the centrifuge.

With the completion of this process, it is possible to observe an orange-colored area in the tube (L-PRF) and the rest of the blood material just below. L-PRF was collected from the tubes (Figure 2).
Volume II Issue 6 - 2020

\section{Priscila Carneiro Oliveira, Beatriz dos Santos Borges, Renatha Oliveira de Souza Moura \\ Master in Periodontics, Specialist in Implantology, UNINASSAU, Brazil}

Correspondence: Priscila Carneiro Oliveira, Master in Periodontics, Specialist in Implantology, UNINASSAU, Brazil, Email carneiropriscila@hotmail.com

Received: October 26, 2020 | Published: December 2I, 2020

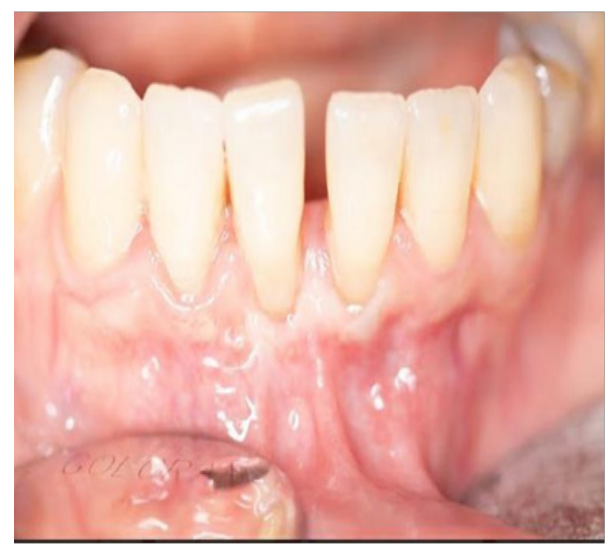

Figure I Miller class I recession.

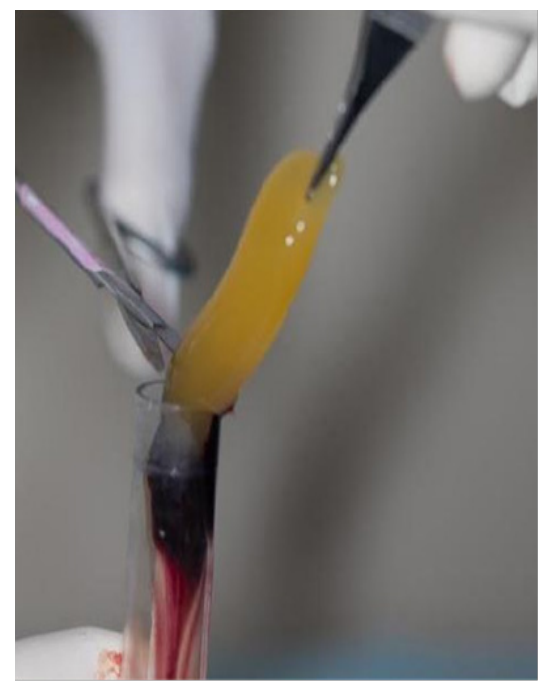

Figure 2 L-PRF collected from the tubes.

The L-PRF was placed in a stainless steel case for PRF and L-PRF (Figure 3), to remove excess liquid, assisting in the handling and application of the Fibrin Rich Plasma. 


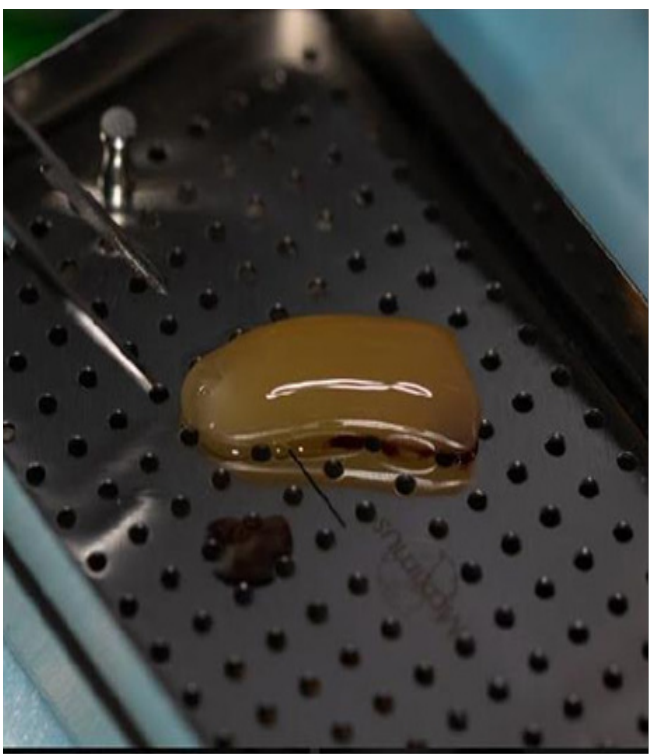

Figure 3 L-PRF in the stell case.

After anesthesia by blocking the lower alveolar nerve and local papillary supplementation with $4 \%$ Articaine with epinephrine 1: 100,000 (Nova DFL ${ }^{\circledR}$ ), the oral environment was adjusted by supragingival scraping with a Gracey 7-8 curette (Hu-Friedy ${ }^{\circledR}$, Chicago) for root planning, removal of the smear layer and other possible contaminants, abundant washing with saline and instructions for oral hygiene.

The recipient bed was prepared with tunneling technique (Figure 4) through intrasucular incisions, creating a supraperiosteal tunnel to accommodate the graft.

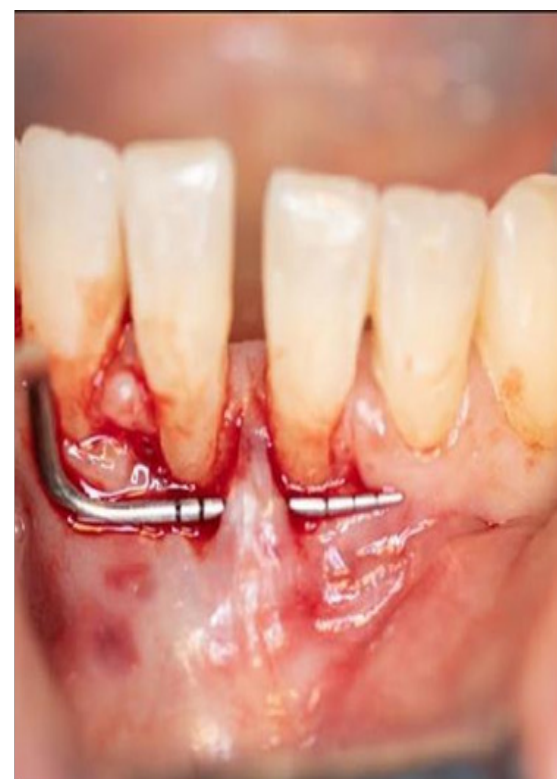

Figure 4 Recipient bed prepared with tunneling technique.

The graft was then positioned on the recipient bed in the cervical region of teeth 31 and 41 and finally sutured. (Figure 5). In order to improve post-operative comfort for the patient, Nimesulide $100 \mathrm{mg}$ (12/12h, for 03 days), Dipyrone Sodium 500mg (06/06h for 3 days) and 24 hours after surgery use of Chlorhexidine Gluconate $0,12 \%$ for the mouthwash.
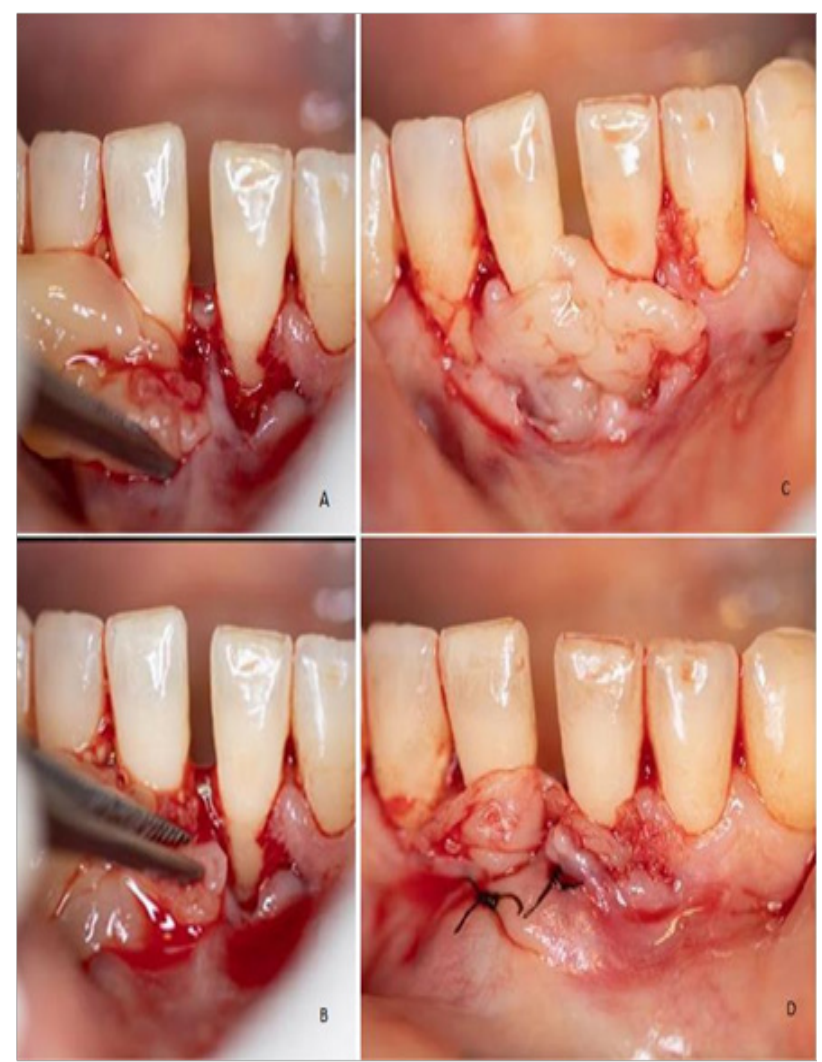

Figure 5 (A) and (B) The L PRF membrane being accommodated in the receiving bed. (C) membrane in position; suture and final clinical appearance.

\section{Discussion}

Marenzi et al. ${ }^{6}$ developed a study to evaluate the efficiency in the healing of soft tissues performed by PRF and leukocytes (L-PRF). The results found at 3 and 7 days revealed a statistically significant improvement in healing for the side that received L-PRF compared to the control side. Thus, the author concluded that the use of L-PRF is efficient for the initial healing process, helping with hemostasis and epithelial closure. ${ }^{6}$

The authors who investigated the use of PRF to treat intraosseous defects do not agree with each other. For Choukroun ${ }^{7}$ the use of PRF is not effective for bone regeneration. ${ }^{7}$ Thorat $^{8}$ report positive results regarding the correction of intra-bone defects when using PRFOs. ${ }^{8}$ Authors Choukroun et al., ${ }^{7}$ Miron et al. as well as Marenzi et al. ${ }^{6}$ agree in their studies that the PRF is efficient in healing soft tissue wounds, obtained favorable results and concluded that the PRF helps in the wound healing process. ${ }^{5,7,8}$ Analysing the positive results in the different applications of L-PRF, the authors Choukroun et al. ${ }^{7}$ stated that the PRF can be considered as a healing biomaterial because it has all the necessary parameters allowing the ideal repair. Agrawal et al. ${ }^{2}$ state that L-PRF, is some material rich in autologous platelets and growth factors, which stimulate the patient's own cells towards a regenerative response. ${ }^{7,9}$

\section{Final considerations}

Based on the literature review and the clinical case report, it was possible to observe that the PRF technique can become a good alternative to replace the techniques that require two surgical sites, in addition to bringing less postoperative discomfort to patients.In order 
for this technique to be even more effective and not cause any further problems, it is essential that the dental surgeon provide oral hygiene and biomechanical control of the biofilm, in addition to removing the etiological factor of the recession.It is understood that further studies are needed, but the technique has shown itself to be very promising.

\section{Funding}

None.

\section{Acknowledgments}

None.

\section{Conflicts of interest}

The authors declare no conflicts of interest related to this study.

\section{References}

1. Takamori ER, Teixeira MVT, Menezes K, et al. A: Platelet-rich fibrin: preparation, definition of quality, clinical use. Visa Magazine in Debate. 2018;6(1):118-124.

2. Agrawal A. A Evolution, current status and advances in application of platelet concentrate in periodontics and implantology. World J Clin Cases. 2020;5(5):159-171.
3. Castro A, Nastaran Meschi, Andy Temmerman, et al. Regenerative potential of leucocyte- and platelet-rich fibrin. Part A: intra-bony defects, furcation defects and periodontal plastic surgery. A systematic review and meta-analysis. J Clin Periodontol. 2017;44(1):67-82.

4. Vasconcellos AVB, Teixeira APE, Cruz PV. Fibrin-rich platelet: a new concept of tissue repair. Innov Magazine. Implant J Biomater Esthet. 2008;3(6):27-31.

5. Rosa PSM. The use of the PRF membrane to replace the free gingival graft in Periodontics. 2019.

6. Mareznzi G, Riccitiello F, Auntie M, et al. Influence of Leukocyte - and Platelet-Rich Fibrin (L-PRF) in the Healing of Simple Postextraction Sockets: A Split-Mouth Study. Biomed Res Int. 2015;2015:369273.

7. Choukroun. J. Platelet - rich fibrin (PRF): A second - generation platelet concentrate. Part V: Histologic evaluations of PRF effects on bone allograft maturation in sinus lift. Oral Surg Oral Med Oral Pathol Oral Radiol Endod. 2006;101(3):299-303.

8. Thorat M, Pradeep AR, Pallavi B. Clinical effect of autologous plateletrich fibrin in the treatment of intra-bony defects: a controlled clinical trial. J Clin Periodontol. 2011;38(10):925-932.

9. Chatterjee A, Agarwal P, Krishna S. Platelet rich fibrin: an autologous bioactive membrane. Elsevier. 2014;11(1):24-26. 